\title{
Criminologie
}

\section{Caractéristiques du fonctionnement social des femmes incarcérées}

\author{
Elham Forouzan, Fanny Malingrey et Sébastien Brouillette-Alarie
}

Volume 45, numéro 1, printemps 2012

URI : https://id.erudit.org/iderudit/1008385ar

DOI : https://doi.org/10.7202/1008385ar

Aller au sommaire du numéro

\section{Éditeur(s)}

Les Presses de l’Université de Montréal

ISSN

0316-0041 (imprimé)

1492-1367 (numérique)

Découvrir la revue

Citer cet article

Forouzan, E., Malingrey, F. \& Brouillette-Alarie, S. (2012). Caractéristiques du fonctionnement social des femmes incarcérées. Criminologie, 45(1), 271-298. https://doi.org/10.7202/1008385ar
Résumé de l'article

La présente étude trace un portrait des caractéristiques du fonctionnement social de 141 femmes incarcérées dans deux établissements provinciaux au Québec. L'ensemble des résultats suggère que non seulement certaines caractéristiques du dysfonctionnement social de ces femmes sont similaires à celles des femmes incarcérées dans d'autres établissements provinciaux au Canada et dans des établissements fédéraux, mais elles semblent présenter aussi certaines caractéristiques particulièrement problématiques pendant leur vie dans la collectivité et ce, dès leur jeune adolescence. Ces résultats suggèrent la pertinence d'élaborer des programmes d'intervention spécifique auprès de ces femmes afin de prévenir leur implication précoce dans un fonctionnement social criminalisé ainsi que le risque de récidive lors de leur retour dans la collectivité. 


\title{
Caractéristiques du fonctionnement social des femmes incarcérées
}

\author{
Elham Forouzan \\ Psychologue clinicienne et chercheure \\ Institut Philippe-Pinel de Montréal \\ Psychologue clinicienne \\ Centre de psychiatrie légale de Montréal \\ elham.forouzan.ippm@ssss.gouv.qc.ca \\ Fanny Malingrey \\ Étudiante au doctorat \\ Faculté de psychologie, Université de Montréal \\ fanny.malingrey@umontreal.ca \\ Sébastien Brouillette-Alarie \\ Étudiant à la maîtrise \\ École de criminologie, Université de Montréal \\ sbalarie@yahoo.ca
}

RÉSUMÉ - La présente étude trace un portrait des caractéristiques du fonctionnement social de 141 femmes incarcérées dans deux établissements provinciaux au Québec. L'ensemble des résultats suggère que non seulement certaines caractéristiques du dysfonctionnement social de ces femmes sont similaires à celles des femmes incarcérées dans d'autres établissements provinciaux au Canada et dans des établissements fédéraux, mais elles semblent présenter aussi certaines caractéristiques particulièrement problématiques pendant leur vie dans la collectivité et ce, dès leur jeune adolescence. Ces résultats suggèrent la pertinence d'élaborer des programmes d'intervention spécifique auprès de ces femmes afin de prévenir leur implication précoce dans un fonctionnement social criminalisé ainsi que le risque de récidive lors de leur retour dans la collectivité.

MOTS-CLÉs - Criminalité des femmes, dysfonction sociale, récidive.

\section{Introduction}

L'état de la littérature scientifique suggère qu'au cours des vingt-cing dernières années, le taux de criminalité des femmes a augmenté dans plusieurs pays (Blanchette et Motiuk, 1996; Teplin et al., 1996 ; 
Blanchette, 1997; Haywood et al., 2000; Bureau of Justice Statistics, 2001; Bell, 2004; Braithwaite et al., 2005; International Center for Prison Studies, avril 2008). De plus, dans certains pays, dont les ÉtatsUnis et le Canada, les femmes constituent une des populations dont le taux de croissance est des plus élevés du milieu carcéral tant fédéral que provincial, dépassant le taux de croissance d'incarcération des hommes (Trevethan, 1999; Harrison et Beck, 2003; Hartwell, 2004; Braithwaite et al., 2005 ; Société de criminologie du Québec, 2007; Lavoie, 2008a). Plus précisément, aux États-Unis, au cours des dix dernières années, le taux d'incarcération des femmes a augmenté plus rapidement que celui des hommes, se situant à 4,9\% comparé à 2,9\% pour les hommes (Snell et Morton, 1994; Harrison et Beck, 2003). Il en est de même au Canada où le pourcentage de femmes faisant face à des accusations a doublé de 1976 à 2001, passant de 9,9\% à 17,6\% des personnes accusées (Statistique Canada, 2006). De plus, les taux de délits violents commis par les femmes deviennent plus prononcés, passant de 25 à 46 par 100000 habitants en 2005. Ces dernières constituent $4 \%$ de la population dans les établissements fédéraux, $6 \%$ des contrevenants dans des établissements provinciaux et $6 \%$ de la population en détention préventive dans l'attente de leur procès (Kong et AuCoin, 2008). Au Québec, les statistiques sur la criminalité en 2004 révèlent qu'au cours des dix dernières années les femmes représentaient un peu plus de $15 \%$ des auteurs présumés d'infractions au Code criminel (Ministère de la Sécurité publique du Québec, 2004) et constituaient $6 \%$ de la population en milieu fermé dans les services correctionnels provinciaux (Ministère de la Sécurité publique du Québec, 2001).

La criminalité des femmes et l'augmentation du nombre de ces dernières dans les milieux carcéraux ont constitué une sérieuse source de préoccupation pour les instances judiciaires, médico-légales et sociales au cours des dernières années dans la plupart des pays. La multiplication d'études internationales portant sur la criminalité féminine révèle l'ampleur de cette croissance ainsi que les conséquences micro et macro-sociales dévastatrices qui en découlent. De même, l'état de la littérature internationale suggère que le fonctionnement social des femmes incarcérées et leurs habitudes de vie nuisent inévitablement et significativement à leur adaptation, réhabilitation et réintégration sociale tant avant qu'après leur(s) incarcération(s) (Young, 1998; Anderson, 2003 ; Lewis, 2006; International Center for Prison Studies, avril 2008). 
La multiplication des études portant sur les caractéristiques et les besoins des femmes incarcérées en établissements fédéraux, de même que l'élaboration de services ciblant leurs besoins spécifiques témoignent de l'intérêt porté à cette population. Cependant, une même attention n'a pas été accordée aux femmes incarcérées dans les milieux provinciaux dont les profils demeurent largement méconnus des instances légales, sociales et psycho-médicales. De ce fait, les options réservées aux femmes, que ce soit en matière d'hébergement, d'évaluation ou de programmes, sont beaucoup moins développées dans les institutions carcérales provinciales que dans les fédérales (Anderson, 2003; International Center for Prison Studies, avril 2008). Et le Canada ne fait pas exception.

Pourtant, au Canada, la plupart des femmes faisant face à des accusations servent leur sentence dans des établissements carcéraux provinciaux et constituent de ce fait une proportion plus importante de la population carcérale provinciale $(6 \%)$ que de la fédérale $(4 \%)$ (Trevethan, 1999; Kong et AuCoin, 2008; Metrac, 2008). Alors que 989 femmes sont sous responsabilité fédérale, incarcérées ou sous surveillance dans la collectivité en 2007 (Bottos, 2007), le milieu carcéral provincial québécois a fait face à 1736 admissions de femmes pour une capacité carcérale de 160 personnes incarcérées en 2005-2006 (Service correctionnel du Canada, 2007). De plus, alors qu'il est généralement présumé que la plupart $(95 \%)$ des femmes incarcérées au provincial présentent une moindre «dangerosité» que celles incarcérées dans des établissements fédéraux en raison de la nature moins violente de leurs délits (Dossier administratif correctionnel de la Direction générale des services correctionnels du Québec, 2008), il est à préciser que $44 \%$ des femmes incarcérées au provincial comparées à $28 \%$ de celles incarcérées au fédéral sont classées comme présentant «un risque élevé de récidive» (Trevethan, 1999). De même, il est rapporté que les femmes incarcérées au provincial sont plus à risque de recourir à des comportements suicidaires comparativement à celles incarcérées au fédéral, ainsi qu'aux hommes incarcérés et à la population en général (Daigle et al., 1999a). Il est aussi rapporté que compte tenu du manque de services, de programmes de traitement et de soins en matière de santé physique et mentale offerts aux femmes incarcérées dans des établissements provinciaux, certaines d'entre elles tendent à demander aux juges une prolongation de leur sentence afin d'être incarcérées dans un établissement fédéral et bénéficier ainsi des divers services et traite- 
ments offerts (Service correctionnel du Canada, 2007). Toutefois, malgré ces indicateurs de l'ampleur et de l'intensité de la détresse des femmes incarcérées dans des établissements provinciaux ainsi que la recommandation par la Loi sur le système correctionnel du Québec (article 21) de mener des études poussées pour déterminer et prendre en compte les besoins propres aux femmes incarcérées sous la juridiction provinciale, les caractéristiques et, de ce fait, les besoins de ces femmes demeurent largement délaissés et méconnus par les autorités concernées.

Compte tenu de cette situation, le but de la présente étude est de tracer un portrait aussi exhaustif que possible des caractéristiques du fonctionnement social d'un échantillon de femmes incarcérées dans deux établissements provinciaux au Québec. Sur cette base, il serait alors possible de permettre une meilleure identification des multiples problématiques sociales présentées par ces femmes, et ce, afin de mettre en place divers services adaptés à leurs besoins et visant à favoriser leur réhabilitation sociale et à réduire le risque de récidive qu'elles présentent.

\section{État de la littérature sur la population féminine carcérale provinciale au Québec}

Alors que la population féminine carcérale provinciale québécoise a fait l'objet de plusieurs projets de recherche, les résultats d'aucune de ces études n'ont permis de fournir un portrait exhaustif des caractéristiques $\mathrm{du}$ fonctionnement social de cette population. Une telle lacune pourrait s'expliquer en partie par certaines limites de ces études, dont principalement la prise en considération d'un éventail restreint des caractéristiques de ces femmes ou une méthode d'évaluation limitée de ces caractéristiques. En effet, trois types d'études semblent avoir été effectués auprès des femmes incarcérées dans des établissements provinciaux au Québec. Un premier type d'études cible un seul aspect problématique spécifique présenté par cette population, tel que les comportements suicidaires (Daigle et al., 1999a; Alarie, 2001), la prostitution (Dufour, 2005) ou l'insertion et le maintien d'emploi (Frigon, 2003 ; Frigon et al., 2003). Plus récemment, un deuxième type d'études a été mené dans le but de déterminer des liens éventuels entre certains problèmes fréquemment présentés par les femmes incarcérées, tels que les comportements violents, et le suicide chez les hommes et les femmes incarcérés (Mailhot, 2002), le degré de risque suicidaire des femmes incarcérées et leur degré d'hostilité (Lefebvre, 2002) ou les liens entre 
les comportements suicidaires et les troubles mentaux graves (Daigle et Côté, 2003). Parallèlement à ces études sur des aspects problématiques spécifiques de la population féminine carcérale provinciale et des liens éventuels entre ces aspects, un troisième type d'études, de nature descriptive, a été réalisé en 2001 dans le cadre d'une vaste consultation par la Société de criminologie du Québec pour la Direction générale des services correctionnels du ministère de la Sécurité publique du Québec afin de mieux connaître des caractéristiques de la clientèle correctionnelle et de mettre sur pied des interventions et des programmes plus adaptés (Robitaille et al., 2002). Les caractéristiques spécifiques des 161 femmes constituant cet échantillon ont été présentées par Boutet, Lafond et Guay (2006). Enfin, certaines caractéristiques des femmes incarcérées dans des établissements provinciaux québécois sont aussi rapportées dans le dossier administratif correctionnel de la Direction générale des services correctionnels du Québec (2008).

L'ensemble des résultats relatifs aux caractéristiques du fonctionnement social des femmes incarcérées dans des établissements carcéraux provinciaux québécois, tel que rapporté dans ces trois types d'études, sont présentés dans le tableau 1 (p. 276).

Malgré leur indéniable pertinence, les études menées auprès des femmes incarcérées dans des établissements provinciaux au Québec révèlent un portrait parcellaire de cette clientèle. En effet, les études ayant été effectuées auprès de cette population se sont limitées à évaluer un nombre restreint de caractéristiques sociodémographiques et à souligner d'éventuels liens entre certains aspects problématiques spécifiques de ces femmes et ce, au détriment d'autres difficultés rencontrées par ces dernières, donnant ainsi lieu à une connaissance et un profil incomplets des caractéristiques sociales problématiques des femmes incarcérées au provincial. Quant aux études de nature descriptive, bien qu'elles révèlent une série d'informations relatives aux caractéristiques sociodémographiques, familiales, scolaires, conjugales et criminelles de ces femmes, elles présentent, pour la plupart, d'importantes lacunes méthodologiques nuisant significativement à la validité de leurs résultats. À titre d'exemple, ces études ne distinguent pas de manière systématique les hommes et les femmes incarcérés dans leurs résultats ou évaluent des caractéristiques d'un échantillon hétérogène, composé de groupes d'individus ayant connu des démêlés judiciaires, mais bénéficiant de diverses mesures légales (incarcération, sursis, etc.). De même, les résultats de certaines de ces études sont issus d'un questionnaire autorévélé dont la validité 
TABLEAU 1 Caractéristiques du fonctionnement social des femmes
sous la juridiction provinciale au Québec

\begin{tabular}{|c|c|c|c|c|}
\hline $\begin{array}{l}\text { Sources/ } \\
\text { caractéristiques }\end{array}$ & $\begin{array}{l}\text { Daigle et al. } \\
\text { (1999a) } \\
\text { Lefebvre (2002) } \\
\text { Alarie (2001) }\end{array}$ & $\begin{array}{l}\text { Mailhot (2002) } \\
\text { Daigle et Côté } \\
\text { (2003) }\end{array}$ & $\begin{array}{l}\text { Boutet et al. } \\
\text { (2006) }\end{array}$ & $\begin{array}{l}\text { Société de } \\
\text { criminologie } \\
\text { du Québec } \\
\text { (2007) }\end{array}$ \\
\hline $\begin{array}{l}\text { Nature de } \\
\text { l'échantillon }\end{array}$ & $\begin{array}{l}\text { Maison Tanguay } \\
\text { et Orsainville } \\
\mathrm{n}=60 \\
\text { Établissement } \\
\text { Joliette } \\
\mathrm{n}=28\end{array}$ & $\begin{array}{l}\text { Maison } \\
\text { Tanguay } \\
n=103\end{array}$ & $\begin{array}{l}\text { Contrevenantes } \\
\text { dans les } \\
\text { services } \\
\text { correctionnels } \\
\mathrm{n}=161\end{array}$ & - \\
\hline Moyenne d'âge & 33 ans & 35,8 ans & 36 ans & 35 ans \\
\hline Francophone & $86,4 \%$ & - & $84,8 \%$ & - \\
\hline $\begin{array}{l}\text { Statut marital: } \\
\text { célibataire }\end{array}$ & - & $52,4 \%$ & $53,8 \%$ & Majorité \\
\hline mère de famille & - & - & $75,5 \%$ & Majorité \\
\hline $\begin{array}{l}\text { Niveau de } \\
\text { scolarité }\end{array}$ & 10,7 ans & 10,8 ans & - & $\begin{array}{l}\text { Sous- } \\
\text { scolarisées }\end{array}$ \\
\hline Sans emploi & - & $73,8 \%$ & $72,3 \%$ & $\begin{array}{l}\text { Peu/pas } \\
\text { d'expérience } \\
\text { de travail }\end{array}$ \\
\hline $\begin{array}{l}\text { Victime de } \\
\text { violence } \\
\text { conjugale }\end{array}$ & - & - & $68,8 \%$ & \\
\hline $\begin{array}{l}\text { Prestations } \\
\text { d'aide sociale }\end{array}$ & - & - & $36,6 \%$ & Majorité \\
\hline $\begin{array}{l}\text { Amis } \\
\text { judiciarisés }\end{array}$ & - & - & $54,1 \%$ & \\
\hline $\begin{array}{l}\text { Antécédents } \\
\text { judiciaires } \\
\text { juvéniles }\end{array}$ & - & - & $20,9 \%$ & \\
\hline $\begin{array}{l}\text { Antécédents } \\
\text { judiciaires } \\
\text { adultes }\end{array}$ & oui & - & $55,3 \%$ & \\
\hline $\begin{array}{l}\text { Antécédents } \\
\text { délictuels } \\
\text { violents }\end{array}$ & - & $\begin{array}{l}19,6 \% \\
(43,3 \% \\
\text { autorévélé) }\end{array}$ & - & \\
\hline $\begin{array}{l}\text { Antécédents } \\
\text { d'incarcération }\end{array}$ & Au moins 2 & - & $54,4 \%$ & \\
\hline $\begin{array}{l}\text { Consommation } \\
\text { psychoactive }\end{array}$ & - & - & $63,3 \%$ & $89 \%$ \\
\hline $\begin{array}{l}\text { Problématique } \\
\text { toxicomanie }\end{array}$ & - & - & - & $48 \%$ \\
\hline
\end{tabular}


des réponses fournies demeure questionnable. Des auteurs soulignent d'ailleurs que «le lecteur devra garder à l'esprit que la grande majorité des données de ce manuscrit proviennent des perceptions et des réponses fournies par la clientèle dans le contexte d'une entrevue. Conséquemment, certaines sont susceptibles de différer de la réalité» (Boutet et al., 2006).

Ainsi, le profil des femmes qui purgent une sentence d'incarcération de moins de deux ans, tel que dégagé par l'état actuel de la littérature scientifique, demeure incomplet et possiblement biaisé en partie par les lacunes méthodologiques mentionnées et par l'absence d'identification des caractéristiques de leur fonctionnement social. Pourtant, ce sont bien les caractéristiques du fonctionnement social de ces femmes qui contribuent à leur incarcération et, éventuellement, à leur récidive délictuelle et à leur implication dans une «carrière» criminelle. Il est d'ailleurs intéressant de constater que ce sont ces aspects du fonctionnement social des femmes incarcérées qui sont pris en considération lors de l'évaluation des besoins et du niveau de risque de récidive qu'elles présentent (voir le Level of Service Inventory Revised et le Level of Service/Case Management Inventory [LS-CMI, Andrews et al., 2004]).

Compte tenu de ces limites et du peu de connaissance des aspects problématiques du fonctionnement social des femmes sous la juridiction provinciale au Québec, le but de la présente étude est de tracer un portrait aussi exhaustif que possible des caractéristiques du fonctionnement social d'un échantillon de femmes incarcérées dans deux établissements provinciaux au Québec, et ce, afin d'évaluer leurs problématiques sociales et favoriser l'élaboration de services adaptés à leurs besoins.

\section{Méthodologie}

\section{Composition de l'échantillon}

L'échantillon de cette étude est constitué de 141 femmes incarcérées dans deux établissements provinciaux québécois, dont l'un à Montréal (Établissement Maison Tanguay) $(\mathrm{n}=100)$ et l'autre dans la ville de Québec (Centre de détention de Québec - Secteur féminin) $(n=41)$.

L'échantillonnage de cette étude a été constitué de façon aléatoire à partir de 1530 personnes distinctes incarcérées pour la période du 1er janvier 2008 au 30 juin 2009 à l'établissement de détention provincial 
pour femmes Maison Tanguay. De ces femmes, 110 ont été sollicitées pour participer au projet de recherche et 100 ont accepté de collaborer. Le taux de refus est ainsi évalué à 9,1\%. À ces 100 participantes s'ajoutent 41 autres femmes incarcérées au Centre de détention de Québec - Secteur féminin, à Québec. Plus précisément, parmi les 273 femmes distinctes incarcérées entre septembre 2008 et juin 2009, 71 ont été sollicitées pour participer au projet de recherche et 54 ont accepté d'y participer. Le taux de refus est ainsi évalué à 23,9\%. Toutefois, trois de ces participantes ont été libérées avant de participer au projet, sept ont été transférées dans d'autres établissements avant d'entamer leur participation dans la présente étude et trois ont été exclues du projet, l'une en raison d'une limite intellectuelle importante, une parce qu'elle présentait un état dépressif majeur et la dernière parce qu'elle n'a pas rempli en entier un des questionnaires (MCMI-I) (Millon, 1983).

Soulignons que les taux de refus des participantes de la présente étude sont moins élevés que les 36,4\% rapportés par Daigle et Côté (2003) lors de leur étude effectuée auprès de 103 femmes incarcérées entre fin janvier et mi-juillet 2001 à l'établissement Tanguay (768 femmes admises, 162 femmes sollicitées, 103 participantes).

Enfin, précisons que parmi les participantes de la présente étude, la plupart $(84,6 \%)$ purgeaient une sentence provinciale de 325,3 jours en moyenne et $15,4 \%$ étaient en attente de leur sentence.

\section{Instruments de mesure}

Dans le cadre de l'évaluation des caractéristiques du fonctionnement social de ces femmes, l'instrument de mesure employé dans ce projet est un protocole d'entrevue composé de questions ouvertes, administré au cours d'une entrevue individuelle semi-structurée entre une psychologue expérimentée et la participante. Cette dernière est incitée à fournir des informations sociodémographiques et des informations relatives à son histoire développementale, son fonctionnement social, dont ses expériences professionnelles, ses sources de revenu, ses relations conjugales et interpersonnelles, ses problèmes de santé physique et mentale passés et actuels, ses habitudes de consommation de substances psychoactives et ses démêlés avec la justice.

Les réponses des participantes à ce questionnaire ont par la suite été jumelées avec les informations disponibles dans leurs divers dossiers correctionnels afin d'assurer la validité des informations qu'elles avaient 
fournies. Les dossiers médicaux, sociaux et administratifs consultés contenaient des informations relatives aux caractéristiques sociodémographiques, aux antécédents judiciaires et aux diagnostics établis par des psychologues, psychiatres, travailleurs sociaux et autres intervenants dans le milieu carcéral. De même, les informations fournies par les participantes ont été comparées et/ou complétées selon les cas par celles du LS-CMI (Andrews, et al., 2004). Le LS-CMI est un instrument actuariel fréquemment utilisé par le personnel du Service correctionnel du Québec et disponible dans les dossiers de la plupart des participantes. Cet instrument a été validé auprès d'adultes et d'adolescents américains et canadiens criminalisés et non criminalisés ainsi qu'auprès de femmes incarcérées (Williams et al., 2009).

En cas de discordance entre les deux sources d'informations (propos de la participante et contenu de ses dossiers institutionnels), les informations disponibles dans les dossiers institutionnels étaient retenues, étant donné qu'il est considéré que de telles informations présentent une plus grande fiabilité comparativement à celles fournies par les participantes (Hare et al., 1991).

\section{Administration des questionnaires et des tests}

Deux psychologues expérimentées auprès de personnes incarcérées ont effectué les entrevues avec les participantes alors qu'un assistant de recherche, étudiant à la maîtrise en criminologie, était chargé de l'informatisation des informations obtenues lors de ces entrevues, ainsi que de l'étude des dossiers institutionnels des participantes.

Afin de procéder à la sélection des participantes, dans un premier temps, l'ensemble du personnel concerné par ce projet à la Maison Tanguay (Montréal) et au Centre de détention de Québec - Secteur féminin (Québec) a été rencontré et sensibilisé aux objectifs de ce projet. Par la suite, une psychologue et une agente correctionnelle travaillant depuis plusieurs années dans chacun de ces établissements ont été chargées de solliciter la participation des détenues au projet. La sélection des participantes a été effectuée au hasard et le seul critère d'exclusion consistait en la présence de troubles mentaux graves au moment de l'entrevue, étant donné que la présence d'épisodes délirants, par exemple, pouvait compromettre le déroulement de l'entrevue. Il est à préciser que les critères d'exclusion ne prenaient pas en considération les différentes mesures correctionnelles (peine d'incarcération de moins 
de deux ans, détention préventive, etc.) compte tenu de l'absence de différence entre les caractéristiques des individus soumis aux diverses mesures correctionnelles (Montegiani et Soucy, 1994). Une fois la participante sélectionnée par l'intervenant, elle était rencontrée et les objectifs du projet lui étaient expliqués. Si la participante manifestait un intérêt à participer au projet, elle était adressée à l'une des deux psychologues responsables du projet pour entamer une entrevue individuelle.

Au commencement de cette entrevue, des feuilles comportant une description détaillée des objectifs et du déroulement du projet étaient distribuées à la participante et lues à haute voix par l'évaluatrice. Après l'accord de la participante pour collaborer à ce projet, un formulaire de consentement était signé par les deux parties concernées, en conformité avec la Loi sur l'accès aux documents des organismes publics et sur la protection des renseignements personnels. Par la suite, la participante prenait part à une entrevue semi-structurée d'une durée d'environ trois heures.

\section{Analyse des résultats}

En raison de la nature exploratoire de la présente étude, les résultats sont présentés sous forme de moyennes ou de pourcentages. Des tableaux thématiques, regroupant les résultats relatifs aux différentes caractéristiques des expériences de vie des participantes, ont été constitués afin de schématiser l'information extraite. Considérant que les données proviennent d'échantillons issus de deux sites géographiques différents, des comparaisons statistiques ${ }^{1}$ ont été effectuées sur certaines variables démographiques (l'âge des participantes, leur origine ethnique, la durée de la sentence ainsi que leurs résultats aux instruments utilisés dans le cadre de ce projet) pour évaluer l'équivalence de chacune des populations carcérales étudiées. Étant donné qu'aucune différence statistiquement significative n'a pu être observée, il a été décidé de combiner les deux échantillons pour obtenir un portrait global de la situation des femmes incarcérées dans ces établissements de détention provinciaux. Afin de ne pas tronquer les résultats, les données manquantes ont été exclues des analyses. Le fait de ne pas répondre à certaines questions d'entrevue, soit par refus, par ignorance ou parce que la question ne s'appliquait pas à leur situation a été considéré comme

1. Le logiciel employé afin de procéder aux différentes analyses statistiques de la présente étude est la version 16,0 de SPSS. 
une valeur manquante. Par conséquent, pour de telles informations, le nombre (n) de participantes (l'unité statistique de base) peut varier selon les informations présentées.

\section{Résultats}

\section{Caractéristiques sociodémographiques}

Le tableau 2 présente les principales caractéristiques sociodémographiques des femmes de l'échantillon. La moyenne d'âge des participantes de cette étude est de 39,23 ans. Les personnes âgées entre 40 et 60 ans représentent $45,4 \%$ des participantes. La plupart des participantes $(91,3 \%)$ sont nées au Québec. Un peu plus de la moitié des répondantes rapportent qu'elles étaient mariées ou vivaient en union de fait au moment de leur prise en charge par les services correctionnels alors que $41,2 \%$ ont répondu avoir été célibataires. La plupart des participantes $(83,7 \%)$ rapportent avoir été sans emploi lors de leur présente prise en charge par les services correctionnels. Enfin, la plupart des participantes purgent en moyenne une sentence de 325,3 jours (tableau 2).

TABLEAU 2

Caractéristiques sociodémographiques

\begin{tabular}{|l|c|c|}
\hline Variables sociodémographiques & $\%$ /Score moyen & n/Écart-type \\
\hline Âge lors de l'entrevue & 39,23 & 64 \\
$\quad$ Âge moyen & 45,4 & 10,96 \\
$\quad 40$ ans et plus & & \\
\hline Lieu de naissance & 91,3 & 126 \\
$\quad$ Province de Québec & & \\
\hline Ethnie: & 92,9 & 131 \\
$\quad$ Caucasienne & 4,3 & 6 \\
Amérindienne & 2,1 & 3 \\
Afro-américaine & 0,7 & 1 \\
$\quad$ Asiatique & & \\
\hline Situation matrimoniale lors de l'incarcération & 56,0 & 79 \\
$\quad$ Mariée/conjointe de fait & 41,2 & 58 \\
$\quad$ Célibataire & 2,8 & 4 \\
$\quad$ Veuve & & \\
\hline Situation professionnelle lors de l'incarcération & 83,7 & 118 \\
$\quad$ Sans emploi & & \\
\hline Statut carcéral & 15,4 & 21 \\
$\quad$ Prévenues & 325,31 & 217,74 \\
$\quad$ Sentence en jours & & \\
\hline
\end{tabular}




\section{Type des délits actuels}

Pour ce qui est des délits commis précédant la prise en charge actuelle des participantes par les services correctionnels, ils les auraient généralement commis durant les six mois précédant l'entrevue à l'origine de la présente étude. Près de deux tiers des répondantes $(65,2 \%)$ auraient commis plus de deux infractions à l'origine de leur actuelle incarcération. Pour la moitié des participantes $(51,1 \%)$, ces infractions concernaient le non-respect des conditions de mise en liberté (principalement une rechute dans la consommation de substances psychoactives ou la fréquentation de milieux ou d'individus criminalisés); pour $40,4 \%$ d'entre elles, des vols; et pour un tiers $(34,0 \%)$, il s'agissait de délits liés aux stupéfiants. De plus, pour plus d'un tiers des participantes $(36,2 \%)$, les infractions à l'origine de leur présente incarcération auraient été de nature violente et consistaient principalement en des voies de fait $(24,1 \%)$. Enfin, deux tiers des participantes admettent avoir commis des délits pour lesquels elles n'ont jamais été sanctionnées, principalement des délits liés aux stupéfiants (36\%) ou des vols sans recours à la violence (32\%) (tableau 3).

TABLEA U 3

Type des délits actuels

\begin{tabular}{|l|r|r|}
\hline Variables & $\%$ & $\mathrm{n}$ \\
\hline Vols & 40,4 & 57 \\
Vols violents & 7,8 & 11 \\
Délits liés aux stupéfiants & 34,0 & 48 \\
Voies de fait & 24,1 & 34 \\
Meurtres & 4,3 & 6 \\
Délits liés aux armes à feu & 1,4 & 2 \\
Délits sexuels & 4,3 & 6 \\
Délits liés à la conduite d'automobile & 6,4 & 9 \\
Fraudes & 21,3 & 30 \\
Non-respect des conditions de mise en liberté & 51,1 & 72 \\
Enlèvements & 5,0 & 7 \\
Incendies & 1,4 & 2 \\
Entraves & 7,8 & 11 \\
Autres & 21,3 & 30 \\
Infractions actuelles violentes & 36,2 & 51 \\
Délit(s) non officialisé(s) & 66,7 & 78 \\
Vols sans violence & 32,0 & 24 \\
Stupéfiants & 36,0 & 27 \\
\hline
\end{tabular}




\section{Cheminement scolaire et professionnel}

La moitié des participantes $(51,8 \%)$ rapportent avoir fourni une performance scolaire médiocre et la plupart d'entre elles $(71,6 \%)$ précisent avoir présenté des difficultés de concentration, d'apprentissage et/ou des troubles du comportement. De même, près des deux tiers des répondantes $(63,8 \%)$ admettent ne pas avoir terminé leurs études secondaires. De plus, la moitié des participantes $(51,1 \%)$ admettent avoir échoué au moins une année scolaire et plus d'un tiers $(39,7 \%)$ de ces femmes précisent avoir présenté des comportements problématiques ayant engendré leur suspension ou leur expulsion scolaire. Enfin, plus de la moitié des participantes rapportent avoir présenté des difficultés relationnelles avec les pairs et l'autorité (58,9\% et $61,7 \%)$ (tableau 4).

TABLEA U 4

Cheminement scolaire

\begin{tabular}{|l|c|c|}
\hline Variables & $\%$ & $\mathrm{n}$ \\
\hline Faible performance scolaire & & \\
Primaire & 29,3 & 41 \\
Secondaire & 50,8 & 66 \\
Primaire ou secondaire & 51,8 & 73 \\
\hline Difficultés d'apprentissage/concentration & & \\
Primaire & 36,0 & 50 \\
Secondaire & 42,6 & 55 \\
Primaire ou secondaire & 44,3 & 62 \\
\hline Difficultés scolaires & & \\
Primaire & 48,6 & 68 \\
Secondaire & 66,7 & 88 \\
Primaire ou secondaire & 71,6 & 101 \\
\hline Scolarité & & \\
Moins d'une 10 ${ }^{e}$ année & 34,8 & 49 \\
Moins d'une 12 ${ }^{e}$ année & 61,0 & 86 \\
Redouble une année ou plus & 51,1 & 72 \\
Expulsion ou suspension & 39,7 & 56 \\
Secondaire non terminé & 63,8 & 90 \\
\hline Difficultés relationnelles & & \\
Pairs & 58,9 & 83 \\
Autorité & 61,7 & 87 \\
\hline
\end{tabular}

Pour ce qui est de leur cheminement professionnel, bien que la plupart des participantes $(89,1 \%)$ rapportent avoir commencé à travailler vers l'âge de 16 ans, plus des deux tiers $(72,3 \%)$ reconnaissent avoir été fréquemment sans emploi et un tiers d'entre elles $(32,6 \%)$ admettent 
n'avoir jamais occupé un même emploi pour une durée de plus d'un an au cours de leur vie adulte. De même, la plupart des participantes $(83,7 \%)$ reconnaissent avoir été sans emploi lors de leur présente prise en charge par les services correctionnels.

Les types d'emploi occupé par les participantes sont diversifiés, non spécialisés et de courte durée. L'emploi le plus fréquemment évoqué concerne le domaine de la restauration/alimentation (25,6\%), suivi de celui des bars/discothèques (12,8\%). Notons également que $21,8 \%$ de ces femmes s'engageaient dans divers domaines d'emplois non spécialisés. De plus, l'engagement des participantes dans le domaine du travail a été caractérisé par de l'instabilité $(65,9 \%)$ ainsi que des difficultés d'adaptation $(78,2 \%)$. En effet, près d'un tiers d'entre elles mentionnent avoir fréquemment vécu des conflits interpersonnels avec leurs collègues ou leurs employeurs $(30,2 \%)$ et près de la moitié de ces femmes admettent avoir entretenu une attitude irresponsable en consommant des substances psychoactives et/ou en commettant des vols sur leur lieu de travail $(46,2 \%)$. La plupart des participantes rapportent avoir généralement quitté leur emploi de leur plein gré $(62,0 \%)$, par manque d'intérêt, lassitude ou une rémunération insatisfaisante, alors que près de la moitié d'entre elles $(48,9 \%)$ admettent avoir été congédiées pour non-respect des règlements professionnels.

Par ailleurs, près de la moitié des participantes $(46,1 \%)$ admettent s'être adonnées à des activités illégales, de danses érotiques ou de prostitution à titre d'emploi au cours de leur vie. De même, la plupart des répondantes $(69,5 \%)$ reconnaissent avoir eu recours principalement à des activités criminelles à titre d'emploi.

Enfin, en ce qui a trait à la situation financière, la plupart des participantes mentionnent avoir bénéficié des prestations du bien-être social au cours de leur vie $(87,9 \%)$ et plus de la moitié d'entre elles rapportent avoir été endettées au moment d'être prises en charge par les services correctionnels (55,3\%) (tableau 5, p. 285).

\section{Caractéristiques des relations conjugales}

La plupart des participantes se définissent d'orientation hétérosexuelle $(85,1 \%)$. Lors de la prise en charge de ces contrevenantes par le Service correctionnel, un peu plus de la moitié d'entre elles (56\%) vivaient une relation conjugale, mais la plupart $(84,4 \%)$ qualifient cette relation d'insatisfaisante. Un peu plus de la moitié des répondantes $(52,8 \%)$ 
TABLEAU 5

\section{Caractéristiques professionnelles}

\begin{tabular}{|l|c|c|}
\hline Variables & \%/Score moyen & N/Écart-type \\
\hline Situation professionnelle & & \\
Sans emploi & 83,7 & 118 \\
Fréquemment sans emploi & 72,3 & 102 \\
Jamais travaillé plus d'un an & 32,6 & 46 \\
1'r emploi avant l'âge de 18 ans $_{\text {Âge moyen lors du 1' emploi }}^{\text {er }}$ & 128 \\
Travail discontinu & 16,1 & 4,34 \\
\hline Domaine d'emploi & 94,3 & 133 \\
Restaurant/alimentation & & \\
Bars/discothèques & 25,6 & 34 \\
Manuel & 12,8 & 17 \\
Bureau & 11,3 & 15 \\
Vente & 7,5 & 10 \\
Danse nue & 6,0 & 8 \\
Prostitution & 29,8 & 42 \\
Recours à des comportements criminels & 38,3 & 54 \\
Instabilité professionnelle & 69,5 & 98 \\
\hline Attitudes et comportements au travail & 65,9 & 75 \\
Conflits avec collègues ou employeurs & & \\
Consommation de substances psychoactives & 30,2 & 38 \\
au travail & 44,3 & \\
Vols au travail & 14,8 & 58 \\
Vols/consommation de substances & & 19 \\
psychoactives au travail & 46,2 & 61 \\
Difficultés d'adaptation & 78,2 & 104 \\
\hline Motifs de départ de l'emploi & 62,0 & 80 \\
Démission & 48,9 & 69 \\
Congédiement & 87,9 & \\
\hline Situation financière & 55,3 & \\
Prestations d'aide sociale & & \\
Dettes & & \\
\hline
\end{tabular}

rapportent avoir entretenu trois relations conjugales ou plus au cours de leur vie et avoir cohabité avec plus de trois conjoint(e)s dans le passé $(52,4 \%)$. La plupart des participantes $(76,6 \%)$ admettent aussi avoir subi de la violence conjugale à un moment de leur vie et/ou reconnaissent avoir vécu avec des conjoints qui présentaient une problématique de toxicomanie $(72,9 \%)$. Toutefois, une minorité d'entre elles $(17 \%)$ rapportent avoir eu recours à des services communautaires offerts aux victimes de violence conjugale et d'avoir été hébergées (13,5\%). Près de la moitié des contrevenantes $(47,5 \%)$ admettent avoir commis l'adultère dans le passé (tableau 6, p. 286). 
TAB LEA U 6

Situation conjugale et maternelle

\begin{tabular}{|c|c|c|}
\hline Variables & $\% /$ Score moyen & n/Écart-type \\
\hline $\begin{array}{l}\text { Statut civil lors de l'incarcération } \\
\text { En couple ou mariée }\end{array}$ & 56,0 & 79 \\
\hline $\begin{array}{l}\text { Orientation sexuelle } \\
\text { Hétérosexuelle }\end{array}$ & 85,1 & 120 \\
\hline $\begin{array}{l}\text { Situation conjugale } \\
3 \text { relations et plus } \\
3 \text { cohabitations et plus }\end{array}$ & $\begin{array}{l}52,8 \\
52,4\end{array}$ & $\begin{array}{l}74 \\
66\end{array}$ \\
\hline $\begin{array}{l}\text { Vie conjugale insatisfaisante } \\
\text { Conjoint violent } \\
\text { Violence conjugale physique } \\
\text { Violence conjugale psychologique/verbale } \\
\text { Toxicomanie partagée dans le couple } \\
\text { Infidèle envers conjoint }\end{array}$ & $\begin{array}{l}84,4 \\
76,6 \\
70,0 \\
62,9 \\
72,9 \\
47,5\end{array}$ & $\begin{array}{r}119 \\
108 \\
98 \\
88 \\
97 \\
67\end{array}$ \\
\hline Recours au foyer pour femmes battues & 17,0 & 24 \\
\hline Séjour centre communautaire & 13,5 & 19 \\
\hline 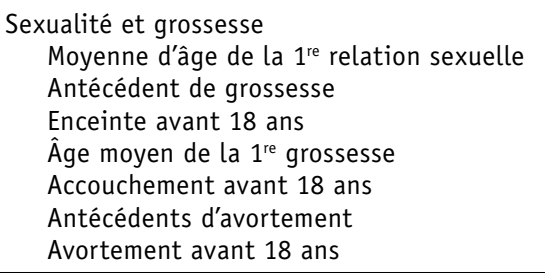 & $\begin{array}{l}14,74 \\
88,7 \\
41,6 \\
18,65 \\
20,8 \\
44,6 \\
20,0\end{array}$ & $\begin{array}{c}2,33 \\
125 \\
52 \\
3,56 \\
26 \\
62 \\
25\end{array}$ \\
\hline $\begin{array}{l}\text { Nombre d'enfants } \\
\text { Plus de } 3 \text { enfants } \\
\text { Sans enfant } \\
\text { Nombre moyen d'enfants }\end{array}$ & $\begin{array}{c}28,4 \\
27,0 \\
1,68\end{array}$ & $\begin{array}{l}40 \\
38 \\
1,49\end{array}$ \\
\hline $\begin{array}{l}\text { Situation des enfants } \\
\text { Enfant placé } \\
\text { Perte de la garde d'un enfant } \\
\text { Enfant placé/garde perdue }\end{array}$ & $\begin{array}{l}60,8 \\
50,0 \\
67,0\end{array}$ & $\begin{array}{l}62 \\
51 \\
69\end{array}$ \\
\hline
\end{tabular}

\section{Grossesse et maternité}

La plupart des participantes $(88,7 \%)$ rapportent avoir été enceintes à un moment de leur vie, dont plus d'un tiers $(38,2 \%)$ avant l'âge de 18 ans. Toutefois, près de la moitié des femmes de l'échantillon $(44,6 \%)$ mentionnent avoir eu recours à l'avortement au moins une fois dans leur vie, dont 20,2\% avant l'âge de 18 ans. La plupart (73,0\%) rapportent avoir un enfant alors que $28,4 \%$ mentionnent en avoir trois ou plus. Toutefois, $67,0 \%$ des participantes précisent ne plus assumer la garde 
de leurs enfants, les ayant placés $(60,8 \%)$ ou ayant perdu leur garde $(50 \%)$ en raison de leur incapacité à assumer leurs responsabilités maternelles (tableau 6).

\section{Caractéristiques du réseau social}

Deux tiers des participantes $(66,0 \%)$ considèrent avoir possédé un réseau social peu développé avant leur incarcération, exempt de liens significatifs d'amitié ou d'activités organisées $(70,9 \%)$. De même, près de la moitié des répondantes $(48,2 \%)$ mentionnent avoir vécu dans un quartier criminalisé et deux tiers $(66,7 \%)$ précisent avoir été entourées de personnes impliquées dans des activités criminelles (conjoint ou membres de la famille). De plus, alors que la plupart des participantes $(89,4 \%)$ admettent aussi avoir entretenu des liens étroits avec des personnes impliquées dans des activités criminelles (connaissances ou amis), plus d'un tiers d'entre elles $(42,6 \%)$ reconnaissent avoir exclusivement fréquenté de telles personnes (tableau 7).

TA B LEA U 7

\section{Caractéristiques du réseau social et des fréquentations}

\begin{tabular}{|l|r|r|}
\hline Variables & $\%$ & $\mathrm{n}$ \\
\hline Réseau social peu développé & 66,0 & 93 \\
Conflits avec parents & 66,7 & 94 \\
Conflits avec fratrie & 57,4 & 81 \\
Aucune activité organisée & 70,9 & 100 \\
Logement quartier criminalisé & 48,2 & 68 \\
Criminalité du conjoint ou de la famille & 66,7 & 94 \\
Connaissances criminelles & 89,4 & 126 \\
Amis criminels & 83,0 & 117 \\
Uniquement des connaissances criminelles & 43,3 & 61 \\
Uniquement des amis criminels & 42,6 & 60 \\
\hline
\end{tabular}

\section{Implication dans des activités criminelles}

La moyenne d'âge des participantes lors de leur première implication dans des activités délictuelles se situe à 26,7 ans et à 30,6 ans pour ce qui est de leurs premiers séjours en milieu carcéral. Une minorité des participantes $(8,6 \%)$ rapportent avoir connu des démêlés judiciaires et avoir été incarcérées $(0,8 \%)$ avant l'âge de 18 ans, tandis que près de 
deux tiers d'entre elles $(63,3 \%)$ mentionnent avoir commis leurs premiers délits et connu leur première incarcération $(59,2 \%)$ alors qu'elles étaient de jeunes adultes (âgées entre 18 et 30 ans). En moyenne, la plupart des participantes de cette étude ont commencé à s'impliquer dans des activités criminelles vers l'âge de 26,7 ans et ont connu leur première incarcération vers 30,0 ans (tableau 8).

TABLEAU 8

Âge lors de l'implication dans des activités criminelles

\begin{tabular}{|l|c|c|}
\hline Variables & \%/Score moyen & n/Écart-type \\
\hline Âge 1 ${ }^{\text {er délit officiel }}$ & & \\
Mineure & 8,6 & 12 \\
Jeune adulte (18-30 ans) & 63,3 & 88 \\
Adulte (31-40 ans) & 14,4 & 20 \\
Plus de 40 ans & 13,7 & 19 \\
Moyenne d'âge du 1 1' délit & 26,71 & 10,38 \\
\hline Âge 1'e incarcération & & \\
Mineure & 0,8 & 1 \\
Jeune adulte & 59,2 & 74 \\
Adulte & 19,2 & 24 \\
Plus de 40 ans & 20,8 & 26 \\
Moyenne d'âge de la 1' incarcération & 30,65 & 11,66 \\
Moyenne d'âge du délit précédent & 35,45 & 8,54 \\
\hline Âge du délit actuel & & \\
Mineure & 0 & 0 \\
Jeune adulte & 25,5 & 36 \\
Adulte & 30,5 & 43 \\
Plus de 40 ans & 44,0 & 62 \\
Moyenne d'âge du délit actuel & 38,60 & 10,19 \\
\hline
\end{tabular}

Antécédents judiciaires et délit(s) actuel(s)

Près de la moitié des participantes $(45,4 \%)$ rapportent avoir eu recours à des activités criminelles dès l'adolescence. Pour 54,7\% d'entre elles, cette implication leur aurait valu des démêlés avec la police. Parmi ces dernières, la plupart (80\%) rapportent avoir fait face à des conséquences légales et posséder un dossier judiciaire juvénile.

Pour ce qui est des antécédents judiciaires à l'âge adulte, la plupart des participantes $(86,5 \%)$ reconnaissent avoir reçu au moins une condamnation, voire plusieurs $(73,8 \%)$ précédant celle à l'origine de leur présente incarcération. De même, 81,9\% d'entre elles mentionnent avoir purgé une peine d'emprisonnement à la suite de ces condamnations. Les délits à l'origine de ces condamnations étaient de nature 
violente pour la moitié des participantes $(59,1 \%)$, principalement des voies de fait $(47,5 \%)$. La plupart des participantes admettent avoir commis des vols $(71,7 \%)$ ou omis de respecter des conditions légales de mise en liberté $(69,2 \%)$. De plus, plus d'un tiers des participantes reconnaissent avoir commis des délits divers (55,0\%).

Enfin, 9,2\% des participantes ayant des antécédents judiciaires mentionnent avoir déjà purgé une peine fédérale (c'est-à-dire une peine d'incarcération de plus de deux ans) avant leur présente prise en charge par les services correctionnels (tableau 9).

TABLEAU 9

Caractéristiques et type des délits antérieurs

\begin{tabular}{|c|c|c|}
\hline Variables & $\%$ & $\mathrm{n}$ \\
\hline Comportements criminels à l'adolescence & 45,4 & 64 \\
\hline Arrestation avant 16 ans & 54,7 & 35 \\
\hline Dossiers juvéniles judiciaires (celles arrêtées) & 80,0 & 28 \\
\hline Dossiers juvéniles judiciaires (ensemble de l'échantillon) & 19,9 & 28 \\
\hline Condamnations antérieures âge adulte & 86,5 & 122 \\
\hline Emprisonnement antérieur à la suite d'une condamnation & 81,9 & 100 \\
\hline Plus d'une condamnation antérieure & 80,1 & 113 \\
\hline Plus de deux condamnations antérieures & 73,8 & 104 \\
\hline Vols & 71,7 & 86 \\
\hline Vols violents & 20,0 & 24 \\
\hline Délits liés aux stupéfiants & 41,7 & 50 \\
\hline Voies de fait & 47,5 & 57 \\
\hline Meurtres & 1,7 & 2 \\
\hline Délits liés aux armes à feu & 5,0 & 6 \\
\hline Délits sexuels & 17,5 & 21 \\
\hline Délits liés à la conduite & 32,5 & 39 \\
\hline Fraudes & 45,8 & 55 \\
\hline Non-respect des conditions de mise en liberté & 69,2 & 83 \\
\hline Antécédents d'évasion & 9,1 & 11 \\
\hline Enlèvements & 0,8 & 1 \\
\hline Incendies & 2,5 & 3 \\
\hline Entraves & 27,5 & 33 \\
\hline Antécédents officiels de voies de fait ou de violence & 59,1 & 71 \\
\hline $\begin{array}{l}\text { Délits divers: vandalisme, troubler la paix, méfait, prostitution, } \\
\text { dommages volontaires, conduire quand permis retiré, suspendu } \\
\text { ou interdit }\end{array}$ & 55,0 & 66 \\
\hline Antécédents de sentence(s) fédérale(s) & 9,2 & 11 \\
\hline
\end{tabular}




\section{Consommation de substances psychoactives}

La plupart des participantes $(86,5 \%)$ admettent une consommation régulière d'une substance psychoactive (alcool ou drogues) au cours de leur vie et la moitié des participantes $(53,9 \%)$ précisent consommer ces deux types de substance régulièrement. Seulement 7,1\% d'entre elles rapportent n'avoir jamais consommé de drogues illicites au cours de leur vie.

Pour la moitié des participantes, le recours à l'alcool a débuté vers l'âge de 14 ans et la consommation de drogues illicites vers l'âge de 16 ans. Toutefois, plus d'un quart des femmes de cet échantillon $(26,2 \%)$ affirment avoir commencé à recourir aux drogues illicites pour la première fois à l'âge adulte.

Par ailleurs, alors que plus de la moitié des participantes (56,7\%) rapportent avoir rencontré des problèmes de nature diverse en raison de leur consommation d'alcool, une proportion moins importante des femmes $(40,4 \%)$ reconnaissent que cette consommation continue d'être un problème dans leur vie. De même, alors que la plupart des participantes $(76,6 \%)$ considèrent que leur consommation de drogues illicites a été à l'origine de leurs problèmes familiaux, conjugaux, financiers ou comportementaux, près des deux tiers $(62,4 \%)$ estiment que le recours à ces substances continue de contribuer à leurs diverses difficultés.

La plupart des contrevenantes de cette étude (57,1\%) expliquent recourir aux substances psychoactives afin de «fuir» la réalité de leur vie alors que près de la moitié des participantes $(47,1 \%)$ reconnaissent rechercher «l'effet excitant» de telles substances. Enfin, deux tiers des participantes $(67,5 \%)$ présentant une problématique de toxicomanie rapportent avoir bénéficié dans le passé de cures de désintoxication, mais plus de la moitié d'entre elles $(54,4 \%)$ reconnaissent ne pas avoir été en mesure de mener à terme ces programmes (tableau 10, p. 291).

\section{Interprétation des résultats et conclusion}

Les résultats de cette étude menée auprès d'un échantillon constitué de 141 femmes incarcérées dans deux établissements carcéraux du Québec suggèrent d'une part l'existence de caractéristiques similaires entre le fonctionnement social des femmes incarcérées sous la juridiction au Québec et de celles incarcérées dans d'autres établissements provinciaux ailleurs au Canada et de celles incarcérées sous la juridiction fédérale. 
TABLEAU 10

\section{Consommation de substances psychoactives}

\begin{tabular}{|c|c|c|}
\hline Variables & $\% /$ Score moyen & n/Écart-type \\
\hline $\begin{array}{l}\text { Âge } 1^{\text {re }} \text { consommation d'alcool } \\
\text { Enfance } \\
\text { Adolescence } \\
\text { Adulte } \\
\text { Moyenne d'âge début consommation } \\
\text { d'alcool (ans) } \\
\text { Aucune consommation d'alcool }\end{array}$ & $\begin{array}{c}24,4 \\
57,8 \\
17,8 \\
\\
14,56 \\
2,1\end{array}$ & $\begin{array}{c}33 \\
78 \\
24 \\
4,68 \\
3\end{array}$ \\
\hline $\begin{array}{l}\text { Âge } 1^{\text {re }} \text { consommation de drogue } \\
\text { Enfance } \\
\text { Adolescence } \\
\text { Adulte } \\
\text { Moyenne d'âge début drogues (ans) } \\
\text { Aucune consommation de drogue } \\
\text { Moyenne d'âge début alcool ou drogue (ans) }\end{array}$ & $\begin{array}{c}24,6 \\
49,2 \\
26,2 \\
16,22 \\
7,1 \\
15,53\end{array}$ & $\begin{array}{c}32 \\
64 \\
34 \\
6,54 \\
10 \\
4,96\end{array}$ \\
\hline $\begin{array}{l}\text { Problème de consommation à l'âge adulte } \\
\text { Alcool } \\
\text { Drogues } \\
\text { Alcool ou drogues } \\
\text { Alcool et drogues } \\
\text { Médication psychotrope } \\
\text { Alcool, ou drogues, ou médication psychotrope }\end{array}$ & $\begin{array}{l}61,7 \\
78,7 \\
86,5 \\
53,9 \\
19,0 \\
87,2\end{array}$ & $\begin{array}{r}87 \\
111 \\
122 \\
76 \\
26 \\
123\end{array}$ \\
\hline $\begin{array}{l}\text { Problème de consommation antérieur } \\
\text { Alcool } \\
\text { Drogues }\end{array}$ & $\begin{array}{l}56,7 \\
76,6\end{array}$ & $\begin{array}{r}80 \\
108 \\
\end{array}$ \\
\hline $\begin{array}{l}\text { Problème actuel de consommation } \\
\text { Alcool } \\
\text { Drogues }\end{array}$ & $\begin{array}{l}40,4 \\
62,4\end{array}$ & $\begin{array}{l}57 \\
88\end{array}$ \\
\hline $\begin{array}{l}\text { Types de problèmes engendrés par la } \\
\text { consommation de substances psychoactives } \\
\text { Infractions à la loi } \\
\text { Conflits familiaux/conjugaux } \\
\text { Difficultés au travail } \\
\text { Aspects médicaux } \\
\text { Autres }\end{array}$ & $\begin{array}{l}78,7 \\
73,8 \\
72,3 \\
35,5 \\
13,5\end{array}$ & $\begin{array}{r}111 \\
104 \\
102 \\
50 \\
19\end{array}$ \\
\hline $\begin{array}{l}\text { Motif de consommation } \\
\text { Fuite réalité } \\
\text { Effet excitant }\end{array}$ & $\begin{array}{l}57,1 \\
47,1\end{array}$ & $\begin{array}{l}68 \\
56\end{array}$ \\
\hline $\begin{array}{l}\text { Traitement des problèmes de consommation } \\
\text { Cure de désintoxication/thérapie spécialisée } \\
\text { Cure de désintoxication menée à terme }\end{array}$ & $\begin{array}{l}67,5 \\
45,6\end{array}$ & $\begin{array}{l}83 \\
36\end{array}$ \\
\hline
\end{tabular}

En effet, tout comme d'autres femmes incarcérées sous la juridiction tant provinciale que fédérale (Blanchette, 1996; Trevethan, 1999; Nicholls et al., juin 2004; Lewis, 2006; Metrac, 2008), la plupart des 
participantes de la présente étude sont sous-scolarisées, sans diplôme d'études secondaires, sans emploi lors de l'incarcération et bénéficiant des prestations de l'aide sociale (Blanchette, 1996; Société de criminologie du Québec, 2007). De même, la plupart d'entre elles sont impliquées dans des relations conjugales caractérisées par la violence et la toxicomanie partagée (Tien et al., 1993; Blanchette, 1996; Laishes, 2002). De plus, la plupart de ces femmes sont mères de famille (Laishes, 2002; Nicholls et al., juin 2004; Boutet et al., 2006; Lewis, 2006; Société de criminologie du Québec, 2007: 156), mais une minorité d'entre elles aurait la garde de son enfant (Nicholls, et al., juin 2004). Enfin, tel que rapporté par d'autres études (Tien et al., 1993; Boutet et al., 2006; Société de criminologie du Québec, 2007; International Center for Prison Studies, avril 2008) les résultats de la présente étude suggèrent que le fonctionnement social de la plupart des femmes incarcérées est affecté par une problématique persistante de dépendance et/ ou d'abus de substances psychoactives et ce, malgré l'engagement de la plupart d'entre elles dans des programmes de désintoxication (Boutet et al., 2006).

D'autre part, les résultats actuels suggèrent qu'en comparaison des résultats de travaux menés auprès de femmes incarcérées dans des établissements provinciaux ailleurs au Canada et de ceux menés auprès de femmes purgeant leur sentence dans des établissements fédéraux canadiens, les participantes de la présente étude se distinguent par plusieurs caractéristiques sociodémographiques, ainsi que par leur fonctionnement social. En effet, presque la totalité de ces participantes sont d'origine caucasienne et québécoise, alors que dans certains établissements provinciaux/territoriaux et fédéraux canadiens, 25 à 30\% des femmes incarcérées sont d'origine autochtone (Kong et AuCoin, 2008). Le faible nombre de femmes aborigènes dans la présente étude pourrait s'expliquer en partie par le nombre plus restreint de la population autochtone au Québec comparé aux autres provinces du Canada. Ces résultats pourraient suggérer que les services et programmes à élaborer auprès des femmes incarcérées dans des établissements provinciaux québécois ne nécessitent pas la prise en compte de différences ethniques, culturelles ou religieuses. Toutefois, d'autres études seront nécessaires afin de déterminer s'il s'agit là d'un résultat représentatif ou non de la population féminine carcérale québécoise. Par ailleurs, les participantes de la présente étude sont plus âgées que d'autres femmes incarcérées tant sous la juridiction provinciale (Daigle et al., 1999a; 
Daigle et al., 1999b; Trevethan, 1999; Alarie, 2001; Lefebvre, 2002; Mailhot, 2002; Daigle et Côté, 2003; Nicholls et al., juin 2004; Société de criminologie du Québec, 2007; Kong et AuCoin, 2008) que fédérale ayant fait l'objet de travaux de recherche antérieurs (Blanchette et Motiuk, 1996; Lewis, 2006; Kong et AuCoin, 2008). Ces résultats pourraient suggérer un effet de vieillissement de la clientèle correctionnelle observable depuis 1986 (Cousineau et al., 1994; Robitaille et al., 2002) puisque la proportion de la clientèle féminine carcérale provinciale ayant plus de 40 ans était de $17,7 \%$ en 1993, de $28,4 \%$ en 2001 et de $45,4 \%$ pour le présent échantillon. Cette moyenne d'âge plus élevée pourrait avoir une pertinence dans la détermination des programmes et services à élaborer puisque les besoins, les ressources et les capacités des femmes incarcérées dans la quarantaine diffèrent considérablement de ceux des femmes incarcérées dans la vingtaine ou la trentaine.

Pour ce qui est des caractéristiques de leur fonctionnement social, alors que celui de la plupart des participantes de la présente étude est imprégné de criminalité, notamment avec la fréquentation de conjoints et d'amis criminalisés, seule une minorité de femmes incarcérées sous la juridiction fédérale posséderaient un réseau social criminalisé (Blanchette et Motiuk, 1996; Lewis, 2006). De même, alors qu'une minorité ou au plus la moitié des participantes d'études menées auparavant dans les mêmes établissements provinciaux (Mailhot, 2002; Daigle et Côté, 2003) et de celles de projets de recherche menés dans d'autres établissements provinciaux et fédéraux (Blanchette et Motiuk, 1996; Nicholls et al., juin 2004; Boutet et al., 2006; Kong et AuCoin, 2008) posséderaient des antécédents d'incarcération et des antécédents de délits violents, la plupart des participantes de la présente étude ont commis des infractions violentes dans le passé et ont effectué des séjours en milieu carcéral.

Enfin, les résultats de cette étude révèlent l'existence d'informations nouvelles et pertinentes relatives au dysfonctionnement social des femmes incarcérées sous la juridiction provinciale au Québec, ce qui pourrait avoir des conséquences significatives sur la nature et le contenu des programmes de prévention et d'intervention à élaborer. En effet, il ressort que ces femmes présentent des difficultés scolaires dès les premières années de leur scolarisation, ces difficultés consistant principalement en une performance médiocre, des échecs répétés, des suspensions et expulsions en raison de troubles du comportement. Ces données supposent donc qu'un dépistage précoce des filles ou 
adolescentes présentant de telles difficultés pourrait en partie diminuer la probabilité que ces dernières abandonnent précocement leurs études. De même, alors que les résultats de la plupart des études menées auprès des femmes incarcérées se limitent à leur occupation professionnelle durant l'année ou les mois précédant leur incarcération, les résultats de la présente étude permettent de préciser que la plupart des femmes incarcérées ayant constitué le présent échantillon sont souvent sans emploi ou occupent un emploi illégal, présentent de l'instabilité, de l'irresponsabilité, des difficultés d'adaptation leur ayant valu des congédiements et des conflits interpersonnels avec des employeurs ou des collègues. Cette précarité sur le plan de l'emploi cause des problèmes financiers importants à la moitié de ces femmes qui reconnaissent être endettées lors de leur incarcération, ce qui signifie ainsi que lors de leur sortie dans la collectivité, elles risquent de se retrouver aux prises avec des problèmes financiers, ce qui augmente les chances d'une nouvelle implication dans la criminalité. Les difficultés d'assumer leurs responsabilités et leurs engagements ainsi que l'incapacité à respecter autrui affectent significativement le fonctionnement de ces femmes tant sur le plan professionnel que dans leur vie conjugale (infidélités, violence, toxicomanie et criminalité partagées) et dans leur rôle de mère (perte de la garde de leur enfant), et de citoyenne (toxicomanie et criminalité persistant de l'adolescence à l'âge adulte).

Dans l'ensemble, les résultats suggèrent que la plupart des femmes incarcérées sous la juridiction provinciale au Québec et ayant participé à la présente étude présentent un fonctionnement social fortement teinté d'irresponsabilité, de manque de considération envers leurs engagements et autrui, ainsi que de criminalité, et ce, dès leur adolescence. Ces résultats suggèrent donc aussi que des programmes d'intervention ciblant spécifiquement les jeunes filles présentant de telles problématiques sociales pourraient constituer des programmes visant à prévenir un dysfonctionnement social persistant et un mode de vie criminalisé à l'âge adulte. De même, le portrait du fonctionnement social dégagé par ces résultats est au moins aussi problématique, sinon plus, concernant certains aspects, que celui des femmes incarcérées sous la juridiction fédérale et pour qui davantage de programmes d'intervention et d'aide sont développés et offerts. Plus encore, alors qu'il est rapporté que, parmi les femmes incarcérées sous la juridiction fédérale et ayant commis des infractions violentes, "les domaines de besoins les plus faibles sont ceux relatifs à leur fonctionnement dans la collectivité» 
(Bottos, 2007), les résultats de la présente étude suggèrent au contraire qu'un nombre significatif de femmes incarcérées sous la juridiction provinciale au Québec présenteraient des besoins significatifs relatifs à leur fonctionnement dans la collectivité, ce qui nécessiterait l'élaboration de programmes et services adaptés à ces besoins spécifiques et ce, afin de réduire le risque de leur implication dans des activités criminelles et la récidive.

\section{Références}

Alarie, M. (2001). Les comportements suicidaires chez les femmes incarcérées. Mémoire non publié. Université du Québec à Trois-Rivières, Trois-Rivières.

Anderson, T. L. (2003). Issues in the availability of healthcare for women in prison, in female prisoners in the United States: Programming needs, availability, and efficacy. In S. Sharp (Ed.). New Jersey: Prentice Hall.

Andrews, D. A., \& Bonta, J. L. (1995). The Level of Service Inventory-Revised. Toronto: Multi-Health Systems.

Andrews, D. A., Bonta, J. L., \& Wormith, J. S. (2004). Level fo service/case management inventory. An offender assessment system. User's Manual. New York: Multi-Health Systems.

Bell, A. (2004). Délinquantes avec antécédents d'infractions violentes: Une comparaison. Forum - Recherche sur l'actualité correctionnelle, 16, 22-24.

Blanchette, K. (1996). The relationships between criminal history, mental disorder, and recidivism among federally sentenced female offenders. Ottawa: Carleton University.

Blanchette, K. (1997). Comparing violent and non-violent female offenders on risk and need. Forum on Corrections Research, 9, 14-18.

Blanchette, K., \& Motiuk, L. L. (1996). Female offenders with and without major mental bealth problem: A Comparative investigation. Ottawa: Correctional Service of Canada.

Bottos, S. (2007). Les femmes et la violence: Théorie, risque et conséquences pour le traitement (No. 2008 No R-198). Ottawa: Service correctionnel Canada.

Boutet, M., Lafond, G., \& Guay, J. P. (2006). Femmes au Canada, Rapport statistique fondé sur le sexe. Disponible sur www.msp.gouv.qc.ca/reinsertion/ publicat/portrait_2001/profil_clientele_correctionnelle/profil_femmes_ contrevenantes.pdf.

Braithwaite, R. L., Treadwell, H. M., \& Arriola, K. R. J. (2005). Health disparities and incarcerated women: A population ignored. American Journal of Public Health, 95, 1679-1681.

Bureau of Justice Statistics (2001). Prevalence of imprisonment in the U. S. Population, 1974-2001. National Criminal Justice Reference Service, www.ncjrs. gov/App/Publications/abstract.aspx ? ID =197976 
Cousineau, M. M., Landreville, P., Laplante, P., \& Michaud, L. (1994). Profil et besoins de la population des établissements de détention. Montréal: Centre International de Criminologie Comparée, Groupe de recherche et d'analyse sur les politiques et les pratiques pénales.

Daigle, M. S., Alarie, P. M., \& Lefebvre, M. (1999a). La problématique suicidaire chez les femmes incarcérées, Forum. Recherche sur l'actualité correctionnelle, $11,41-45$.

Daigle, M. S., \& Côté, G. (2003). Troubles mentaux et problématique suicidaire chez les femmes incarcérées dans un établissement de détention provincial. Disponible sur: Ministère de la sécurité publique du Québec, www.crise.ca/fr/e-docs/ daigle2003_femmes.pdf.

Daigle, M. S., Lefebvre, P., \& Alarie, M. (1999b). Auto et hétéro-agressivité chez les femmes incarcérées. Annales de l'ACFAS 1999. Disponible sur www. acfas.ca/congres67/s316.htm

Dossier administratif correctionnel de la Direction générale des services correctionnels du Québec (2008). Disponible sur www.gdddq.org/dossiers/ detention/femme_et_santee.htm

Dufour, R. (2005). Aider les femmes prostituées à se situer au coeur de leur vie. Éditions Multimondes. Disponible sur www.multimondes.qc.ca

Frigon, S. (2003). Homicide conjugal au féminin: d'bier à aujourd'bui. Montréal: Les éditions du Remue-ménage.

Frigon, S., Strimelle, V., \& Renière, C. (2003). Insertion et maintien en emploi des femmes judiciariées du Québec. Comité aviseur pour la clientèle judiciarisée adulte et Emploi Québec.

Hare, R. D., Hart, S. D., \& Harpur, T. J. (1991). Psychopathy and the DSM-IV criteria for antisocial personality disorder. Journal of Abnormal Psychology, 100, 391-398.

Harrison, P. M., \& Beck, A. J. (2003). Prisoners in 2002, Bureau of Justice Statistics. Washington, DC.

Hartwell, S. W. (2004). Comparison of offenders with mental illness only and offenders with dual diagnoses. Psychiatric Services, 55, 145-150.

Haywood, T. W., Kravitz, H. M., Goldman, L. B., \& Freeman, A. (2000). Characteristics of women in jail and treatment orientations: A review. Behavior Modification, 24, 307-324.

International Center for Prison Studies (Avril 2008). International profile of women's prisons. Disponible sur www.hmprisonservice.gov.uk/assets/

Kong, R., \& AuCoin, K. (2008). Female offenders in Canada (No. 85-002-XIE). Ottawa: Canadian Centre for Justice Statistics.

Laishes, J. (2002). The 2002 mental health strategy for women offenders. Disponible sur www.csc-cc.gc.ca/text/prgrm/fsw/mhealth/8_e.shtml

Lavoie, M. C. (2008a). Femmes et justice. Femmes, crimes et détention. Le Bulletin, 9, 13-14.

Lavoie, M. C. (2008b). Femmes et justice. Femmes détenus et santé. Le Bulletin, $10,10-11$. 
Lefebvre, P. (2002). L'hostilité et le risque suicidaire chez les femmes incarcérées. Mémoire non publié. Université du Québec à Trois-Rivières, Trois-Rivières.

Lewis, C. (2006). Treating incarcerated women: Gender matters. Psychiatric Clinical North American, 29, 773-789.

Mailhot, S. (2002). Les gestes violents et le suicide chez les hommes et les femmes incarcérés. Mémoire non publié. Université du Québec à Trois-Rivières, Trois-Rivières.

Metrac (2008). Women in provincial institutions. July 2008. Disponible sur www. owjn.org/owjn_2009/index.php? option=com_content $\&$ view $=$ article $\&$ id $=60$ \&Itemid $=67$.

Millon, T. (1983). Millon clinical multiaxial inventory manual. Minneapolis: Interpretative Scoring Systems.

Ministère de la sécurité publique du Québec (2001). Statistiques 2000 sur la criminalité au Québec. Québec: Direction générale des affaires policières et de la prévention de la criminalité.

Ministère de la sécurité publique du Québec (2004). Statistiques sur la criminalité au Québec en 2004. Faits saillants. Disponible sur www.msp.gouv.qc.ca

Montegiani, M., \& Soucy, N. (1994). Portrait de la clientèle correctionnelle du Québec 1993. Québec: Direction générale des services correctionnels, Ministère de la Sécurité publique.

Nicholls, T. L., Brink, J., \& Webster, C. D. (Juin 2004). On making progress in understanding psychopatby and violence in women: A critique of the Correctional Service of Canadd's halt of psychopathy research with women. Paper presented at the La femme fatale: Issues in conceptualization and assessment of psychopathy in women, Stockholm, Sweeden.

Robitaille, C., Guay, J.-P., \& Savard, C. (2002). Portrait de la clientèle correctionnelle du Québec 2001. Montréal: Société de criminologie du Québec pour la DGSC du ministère de la Sécurité publique du Québec.

Service correctionnel du Canada (2007). Ten-year status report on women's corrections 1996-2006. Disponible sur www.csc-scc.gc.ca/text/prgrm/fsw/wos24/ index-eng.shtml

Snell, T. L., \& Morton, D. C. (1994). Women in prison: Survey of state prison inmates 1991. Washington, DC: US Department of Justice, Bureau of Justice Statistics.

Société de criminologie du Québec (2007). Aperçu de la situation des femmes incarcérées au Service correctionnel du Québec, 2005-2006. Disponible sur www. societecrimino.qc.ca/actescongres/pdf/bloc_c_atelier_15.pdf.

Statistique Canada (2006). Femmes au Canada. Rapport statistique fondé sur le sexe $\left(5^{\mathrm{e}}\right.$ ed.). Ottawa: Ministère de l'Industrie.

Teplin, L. A., Abram, K. M., \& McClelland, G. M. (1996). Prevalence of psychiatric disorders among incarcerated women, I: Pretrial jail detainees. Archives of General Psychiatry, 53, 505-512.

Tien, G., Lamb, D., Bond, L., Gillstrom, B., \& Paris, F. (1993). Report on the needs assessment of women at Burnaby correctional centre for women. British Colombia: Institute on Family Violence. 
Trevethan, S. (1999). Les femmes incarcérées dans des établissements fédéraux, provinciaux ou territoriaux. Forum-Recherche sur l'actualité correctionnelle, 11, 9-12.

Williams, K., Andrews, D. A., Bonta, J. L., Wormith, J. S., Guzzo, L., \& Brews, A. (2009). The level of service/case management inventory (LS/CMI): Reliability and validity in female offenders. Paper presented at the Annual meeting of the American Psychology - Law Society, TBA, San Antonio, TX.

Young, D. (1998). Health status and service use among incarcerated women. Family Community Health, 21, 16-31.

ABSTRACT - This study characterizes the social functioning of 14 women incarcerated in two provincial correctional institutions in Quebec. The results suggest that these women share some markers of social dysfunction with women in other federal and provincial correctional institutions in Canada, and that their life in the community is problematic even in early adolescence. It would therefore be useful to develop specific intervention programs for incarcerated women that would prevent their early involvement in criminalized social environments and reduce their risk of recidivism upon return to life in the community.

KEYWORDS • Female criminality, Social dysfunction, Recidivism.

RESUMEN - El presente estudio bosqueja las características del funcionamiento social de 141 mujeres encarceladas en establecimientos provinciales de Quebec. El conjunto de resultados sugiere que no solamente algunas de las características del disfuncionamiento social de estas mujeres son similares a los de las mujeres encarceladas en otros establecimientos provinciales de Canadá y de instituciones federales, sino que parecen presentar también ciertas características particularmente problemáticas en su vida en la comunidad, y ello desde su primera juventud. Estos resultados sugieren la pertinencia de desarrollar programas de intervención específicos para estas mujeres con el fin de prevenir su participación precoz en un funcionamiento social criminalizado, lo mismo que el riesgo de reincidencia una vez de retorno en la colectividad.

PALABRAS CLAVE - Criminalidad femenina, disfuncionamiento social, reincidencia. 